\title{
Dynamics of surface steps
}

\author{
F. Szalma†‡, W. Selke†, and S. Fischer $\dagger$ \\ $\dagger$ †nstitut für Theoretische Physik, Technische Hochschule, D-52056 Aachen, \\ Germany \\ $\ddagger$ Institute for Theoretical Physics, Szeged University, H-6720 Szeged, Hungary
}

\begin{abstract}
In the framework of SOS models, the dynamics of isolated and pairs of surface steps of monoatomic height is studied, for step-edge diffusion and for evaporation kinetics, using Monte Carlo techniques. In particular, various interesting crossover phenomena are identified. Simulational results are compared, especially, to those of continuum theories and random walk descriptions.
\end{abstract}

Key words: SOS model, surfaces, step fluctuations, Monte Carlo simulations PACS: 05.10.Ln, 05.20.Dd, 68.35.Ja

\section{Introduction}

In recent years, the dynamics of steps of monoatomic height on crystal surfaces has attracted much interest, both experimentally and theoretically. Experimentally, equilibrium step fluctuations of isolated steps as well as steps on vicinal surfaces have been studied extensively, following pioneering measurements of $\mathrm{Au}(110)$ [1] and $\mathrm{Cu}(11 \mathrm{n})$ [2] surfaces. The step fluctuations are quantified by the equilibrium function $G(t)=\left\langle\left(h\left(i, t+t_{e q}\right)-h\left(i, t_{e q}\right)\right)^{2}\right\rangle_{i, t_{e q}}$, where $h(i, t)$ denotes the position (or displacement) $h$ of the step at site $i$ and time $t$; one averages over reference times $t_{e q}$ in equilibrium and over the step sites. Typically, the experimental data could be fitted rather well by a power law, $G(t) \propto t^{\alpha}$, with the exponent $\alpha$ being between about $1 / 4$ and about $1 / 2$ [1-4] (attention may be drawn to a discussion on possible ambiguities in determining the intrinsic step fluctuations when using scanning tunneling microscopy [5]).

Theoretically, distinct atomic mechanisms driving the step dynamics have been identified, leading, at long times, to power laws with $\alpha$ being, indeed, 
$1 / 4$ and $1 / 2$ in the limiting cases of step-edge diffusion and evaporationcondensation kinetics, respectively. The scenarios have been established in Langevin (or continuum) [6-8] and other phenomenological [9] descriptions as well as in simulations on SOS models $[6,10,11]$ (for the experimental relevance of the SOS model see, e.g., Ref. 12). In addition to $G(t)$, the corresponding non-equilibrium function $w(t)$ has been analysed, with $w(t)=\left\langle\left(h(i, t)^{2}\right\rangle_{i}\right.$ being the step fluctuations of an initially straight step, say, $h(i, t=0)=0 . w(t)$ is believed to show the same characteristic power-law at late times as $G(t)$. Note that in the case of evaporation-condensation kinetics, an exact solution for $w(t)$ for the closely related discrete one-dimensional Gaussian model, applicable to isolated steps, with arbitrary number of sites is available [13].

In this article, we shall consider step-edge diffusion and evaporation kinetics of isolated and pairs of steps in the framework of SOS models.

For isolated steps, effects of the step length, boundary conditions (periodic and pinned boundary conditions) and temperature are studied systematically, using Monte Carlo techniques, computing both equilibrium, $G(t)$, and nonequilibrium, $w(t)$, step fluctuations. Nontrivial and novel features at early and intermediate times as well as effects due to the finite length of the steps will be emphasized.

For pairs of steps, the role of entropic repulsion on both types of dynamics will be elucidated. Studying various step quantities and correlation functions, intriguing crossover effects are identified, and our simulational results will be discussed in the context of continuum theories and random walk descriptions.

The article is organized accordingly. In the next chapter, we shall present our simulational findings on isolated steps, followed by the chapter on pairs of steps. Each chapter is subdivided in discussing first evaporation kinetics and then step-edge diffusion. A short summary will conclude the paper.

\section{Isolated steps}

A surface step of monoatomic height may be described by the one-dimensional SOS model, defined by [14]

$$
\mathcal{H}=\epsilon \sum_{\langle i, j\rangle}|h(i, t)-h(j, t)|
$$

$h(i, t)$ is the step position at step site $i$ and time $t$; the sum runs over neigh- 
bouring step sites $i$ and $j, j=i \pm 1$. For a step with $L$ active sites, $i$. e. a step of length $L$, the ends of the step may be either pinned, for instance, $h(0, t)=h(L+1, t)=0$ for pinning at equal step positions, or they may be connected by periodic boundary conditions, $h(0, t)=h(L, t)$ and $h(L+1, t)=h(1, t)$ (we set the lattice constant equal to one, with $i$ and $h(i, t)$ being integers). The time is measured in Monte Carlo attempts (MCA) or Monte Carlo steps per site (MCS), with $1 \mathrm{MCS}=L \mathrm{MCA}$.

A pair of steps may be described by two SOS models with the step positions $h_{1}(i, t)$ and $h_{2}(i, t)$, where the subscript 1 refers to the, say, left step and the subscript 2 to the right one. To avoid a double step or crossing of the two steps, one assumes $h_{2}(i, t)>h_{1}(i, t)$, leading to 'entropic repulsion' [15].

Step fluctuations, quantified by the equilibrium function $G(t)$ or the nonequilibrium function $w(t)$, result from the detachment or attachment of an atom at site $i$, decreasing or increasing the step position by one. We shall consider two types of dynamics: (i) a step atom is moved to a neighbouring site, i.e. a detachment at site $i$ is followed by an attachment at site $i \pm 1$ (stepedge diffusion, s-d), and (ii) attachment and detachment events at the step are uncorrelated (evaporation-condensation kinetics, e-c). The probability of accepting the elementary move may be given by the Boltzmann factor of the energy change needed to execute that move, see Eq. (1), implying Glauber kinetics in the case of $\mathrm{e}^{-} \mathrm{c}$ and Kawasaki kinetics in the case of $\mathrm{s}^{-} \mathrm{d}$ [16]. Of course, other types of dynamics may be imagined, for instance, terrace diffusion $[6,8,11]$, but they are outside the scope of this study.

The step fluctuations may then be easily computed in Monte Carlo simulations. To calculate the non-equilibrium function $w(t)$, one averages over an ensemble of $N$ realizations (using different sets of random numbers), starting each time from the initial step configuration, say, $h(i, t=0)=0$. In determining $G(t)$, one first has to equilibrate the step, choosing a sufficiently large reference time, say, $\tau_{0}$. One may then generate a set of successive reference configurations, $h\left(i, \tau_{m}\right)$, with $\tau_{m}=\tau_{0}+m \delta t, m=0,1,2, \ldots M$, $\delta t$ being a constant. $G(t)=\left\langle\left(h\left(i, \tau_{m}\right)-h\left(i, t+\tau_{m}\right)\right)^{2}\right\rangle_{i, \tau_{m}}$ is obtained from averaging over $M$ reference times $\tau_{m}$ and over the step sites $i$; it can be determined in a single, long Monte Carlo run ('dynamic averaging' [11]). When taking into account only one fixed reference equilibrium configuration, $h\left(i, \tau_{0}\right)$, and averaging over an ensemble of realizations (similar to the computation of $w(t)$ ), correlations will usually depend strongly on the choice of the reference configuration.

To obtain accurate and reliable Monte Carlo data, rather extensive sampling 
and care in choosing a suitable random number generator (for instance, the linear congruential random number generator may lead to erroneous results) are needed.

In the following of this chapter, we shall summarize our main simulational findings on isolated steps, with comparisons to results on related exactly solved continuum or discrete cases $[7,13]$. Typically, in the simulations steps with up to 128 sites have been studied.

At very early times, step fluctuations are diffusive [7,13], due to excitations at independent, equivalent step sites, i.e. $w$ (or $G) \propto t$. The diffusion coefficients in the non-equilibrium case are $D_{1, e c}^{w}=\exp \left(-2 \epsilon / k_{B} T\right)$ for e-c, and $D_{1, s d}^{w}=2 \exp \left(-4 \epsilon / k_{B} T\right)$ for s-d. The corresponding diffusion coefficients for the equilibrium function $G$ are somewhat larger, with the enhancement factor tending to increase when lowering the temperature. Strictly speaking, the perfectly diffusive behaviour holds, $L>1$, only in the first Monte Carlo attempt. Already the second attempt leads to either subdiffusive or superdiffusive fluctuations. Accordingly, the effective exponent, e. g. for $w$,

$$
\alpha_{\mathrm{eff}}^{w}(t)=\ln \left(w\left(t_{i+1}\right) / w\left(t_{i}\right)\right) / \ln \left(t_{i+1} / t_{i}\right)
$$

where $t=\sqrt{t_{i+1} t_{i}}$, is then either smaller or larger than one. A simple calculation shows that $w$ becomes superdiffusive in the e-c case (in the second Monte Carlo attempt, there are local step moves, which cost no energy, at sites next to the initial step excitation), while it becomes subdiffusive in the $\mathrm{s}-\mathrm{d}$ case (where the step moves in the second attempt are on average energetically more costly than in the first move). In contrast, the equilibrium step fluctuations $G(t)$ tend to be subdiffusive in both cases.

(i) For evaporation-condensation kinetics, $w^{e c}$ continues to be superdiffusive at early times before crossing over to the subdiffusive regime (see Fig. 1), where the step fluctations are governed by the line tension, approaching, at sufficiently long times for sufficiently long steps the form $w^{e c} \propto t^{1 / 2}$ (with the crossover time increasing like $\left.L^{2}\right)[6,7,13]$. Similar properties hold for $G^{e c}$.

For finite steps, applying pinned (pd) boundary conditions, $w^{e c}$ will eventually reach the saturation value, $w_{\infty}^{e c}=w^{e c}(t \longrightarrow \infty)$, with the effective exponent $\alpha_{\text {eff }}^{w}(t)$ decreasing rather sharply towards zero when approaching saturation at a time being again proportional to $L^{2}$ (see also Fig. 2 and Ref. 13). For sufficiently long steps, we find that $w_{\infty, p d}^{e c}(L)$ approaches the continuum expression 


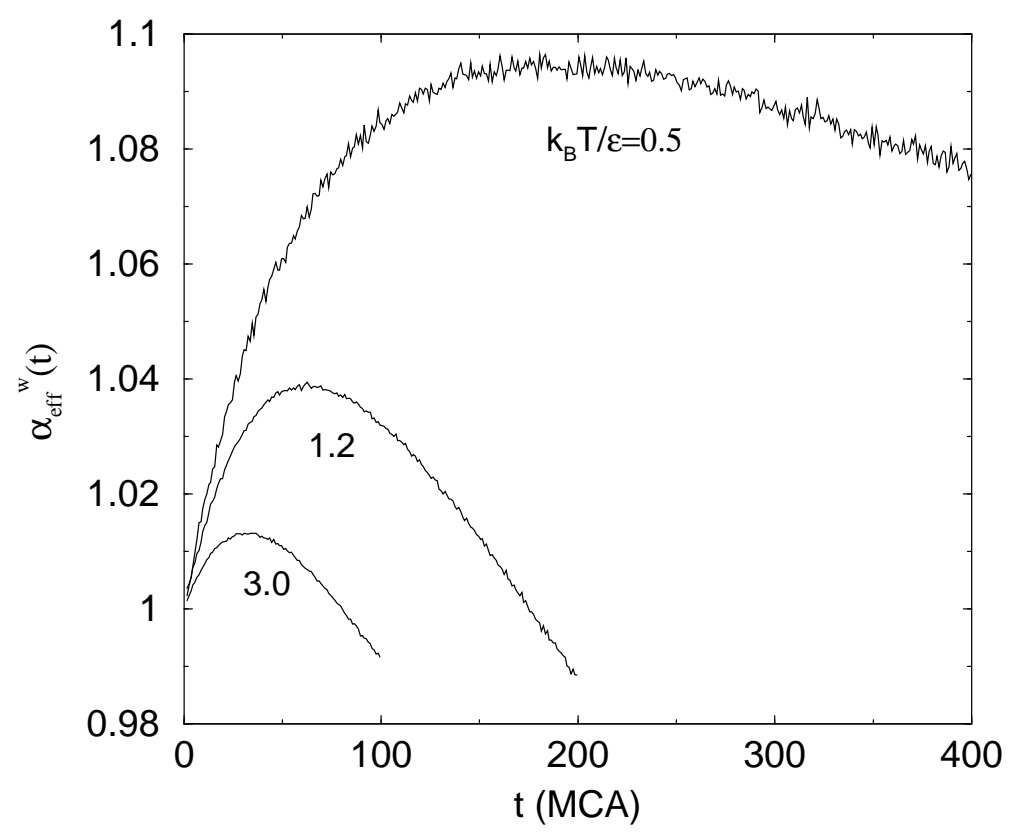

Fig. 1. Effective exponent $\alpha_{\text {eff }}^{w}$ of the non-equilibrium fluctuations of an isolated step, showing the superdiffusive behaviour at early times, when using evaporation kinetics. A step of length $L=128$, at various temperatures, with periodic boundary conditions, was simulated, averaging over $10^{7}$ realizations.

$[7]$

$$
w_{\infty, p d}^{e c}(L)=\frac{1}{6} \frac{k_{B} T L}{\Sigma}
$$

with the step stiffness $\Sigma=2 k_{B} T \sinh ^{2}\left(\epsilon / 2 k_{B} T\right)[17,18]$ (here, attention may be drawn to the recent discussion on the experimental determination of the step stiffness [19]). For short steps, the length dependence of the saturation value is observed to deviate somewhat from the above linear $L$-dependence, due to lattice effects. Note that $G_{\infty, p d}^{e c}(L)=2 w_{\infty, p d}^{e c}(L)$.

In marked contrast, when applying periodic boundary conditions, the time regime where the growth of the step fluctuations is governed by the line tension, $w^{e c} \propto t^{1 / 2}$, is followed by a regime in which the step fluctuations are dominated by the diffusive motion of the roughened step. The entire step now acts like a random walker, with the diffusion coefficient $D_{2 e}^{w}$ approaching $a / L$ for large $L$, where $a$ increases with temperature (similarly for $G$ ). The crossover time to the diffusive behaviour scales like $L^{2}$ [13].- Obviously, at $T=\infty$, each Monte Carlo attempt will be successful, and one always encounters the initial diffusive behaviour of independently and randomly moving single step sites, $D_{1}$. 
(ii) For step-edge diffusion, applying pinned or periodic (pbc) boundary conditions, the step fluctuations are bounded because of the conservation of the average step position in each move. The saturation value of $w^{s d}$ is closely related to that in the e-c case for pinned boundary conditions, see Eq. (3). We find $w_{\infty, p d}^{e c} \approx(5 / 2) w_{\infty, p d}^{s d} \approx(4 / 5) w_{\infty, p b c}^{s d}$ for large $L$. For both boundary conditions, pd and pbc, $G_{\infty}^{s d}(L)=2 w_{\infty}^{s d}(L)$. The time needed to approach the saturation value scales like $L^{4}$. The asymptotic behaviour for indefinitely extended, $L \longrightarrow \infty$, steps, $w, G \propto t^{1 / 4}[6-10]$, is approached closely for finite steps at intermediate times. At earlier times, using periodic boundary conditions, the effective exponent displays an additional interesting non-monotonic behaviour [10], both for $w^{s d}$ and $G^{s d}$. As $L$ increases, the corresponding maximum in $\alpha_{\text {eff }}^{w, G}$ shifts to larger times (the shift being, possibly, proportional to $L^{4}$ ), and it gets weaker and weaker, approaching $1 / 4$ from above. The maximum occurs in $G^{s d}$ about two times later than in $w^{s d}$. For pinned boundary conditions, the corresponding effective exponents, for $w^{s d}$ and $G^{s d}$, decay monotonically in time.

\section{Pairs of steps}

For pairs of steps, we studied both types of kinetics, evaporation-condensation and step-diffusion, applying especially periodic boundary conditions.

(i) In the e-c case, we assumed the two steps of length $L$ to be initially straight, $h_{1(2)}(i, t=0)=h_{1(2)}^{(0)}$, and separated by $d_{0}$ lattice units, i.e. $h_{2}^{(0)}-h_{1}^{(0)}=d_{0}$.

In the simulations, the step length, $1 \leq L \leq 128$, the initial separation distance, $2 \leq d_{0} \leq 60$, and the temperature were varied.

Typical features of the dynamics of the steps are the early superdiffusive behaviour, the step meandering and step roughening driven by line tension, the saturation of fluctuations due to the finite length of the steps, the diffusive motion of each of the roughened steps, and, in addition, phenomena resulting from collisions between the wandering steps. To monitor these aspects, we recorded the non-equilibrium fluctuations of each step, $w_{1}^{e c}(t)=w_{2}^{e c}(t)=w(t)$, the (squared) width of each step $b_{k}^{e c}(t), k=1,2$,

$$
b_{k}^{e c}(t)=\left\langle\left(h_{k}\left(i_{0}, t\right)-h_{k}\left(i_{c}, t\right)\right)^{2}\right\rangle
$$

where $i_{0}$ refers to the first, $i_{0}=1$, or last, $i_{0}=L$, step site, and $i_{c}$ refers to the center site of the step, with $b_{1}^{e c}(t)=b_{2}^{e c}(t)=b(t)$, as well as the average 
distance $d(t)$ between the two steps

$$
d(t)=\frac{1}{L} \sum_{i}\left\langle h_{2}(i, t)-h_{1}(i, t)\right\rangle
$$

The brackets \langle\rangle in the definitions for $b(t)$ and $d(t)$ denote ensemble averages, i.e. averages over $N$ Monte Carlo runs with different random numbers.

By choosing suitable values for the step length $L$, the initial separation distance $d_{0}$, and the temperature $k_{B} T / \epsilon$, typical features of the step dynamics may be disentangled clearly, as exemplified in Fig. 2, showing the time dependence of the effective exponent $\alpha_{\text {eff }}$ of $w, b$, and $d$. There, with $L=128$, $d_{0}=60$, and $k_{B} T / \epsilon=1.0$, one can identify four characteristic successive times, see Fig. 2: $t_{1}$ denotes the time after which step fluctuations approach closely the simple law $w(t) \approx c\left(t+t_{c 1}\right)^{1 / 2}$ (that law is consistent with the asymptotics for indefinitely long isolated steps $w \propto t^{1 / 2}$; most of the deviation of $\alpha_{\text {eff }}^{w}$ from $1 / 2$, as depicted in Fig. 2, may be attributed to the constant $t_{c 1}$ ). At time $t_{2}$, the width $b(t)$ of each step begins to saturate, as reflected by the pronounced decrease in the corresponding effective exponent $\alpha_{\mathrm{eff}}^{b}$. The diffusive motion of the entire, roughened step, its width now being fully saturated, starts to dominate the step fluctuations, $w(t)$, at time $t_{3}$; then $w(t) \approx D_{2 e}^{w} t+t_{c 2}$ (in that purely diffusive regime at times $t>t_{3}$ the effective exponent of $w$ may still be significantly smaller than one because of the constant $t_{c 2}$ ). The first collision between the two wandering steps is indicated by $t_{4}$. The collisions, resulting in the entropic repulsion between the steps, give rise to the increase in the average separation distance $d(t)$, and, at the same time, they lead to a slowing down of the step fluctuations, as measured by $w$. As for two non-crossing usual random walkers in one dimension (corresponding to the case $L=1$ ), perfectly diffusive behaviour, with the diffusion coefficient $D_{2}$ of the isolated steps, shows up again asymptotically at later times. Thence, $\alpha_{\text {eff }}^{w}$ displays a dip after the first collision time $\left(t_{4}\right)$, see Fig. 2, both for pairs of steps and two random walkers, $L=1$.

Of course, for other step parameters the characteristic crossover times may be less clearly separated, and/or more difficult to identify. For instance, when reducing the initial distance between the steps, $d_{0}$, or increasing the length of the steps, collisions between the steps may occur already much earlier than in the example discussed above. In that case, the separation distance $d(t)$ is expected to grow like $t^{1 / 4}$ due to the entropic repulsion during the roughening of the two steps [20]. We simulated such situations as well. In any event, at late stages, one eventually encounters, for steps of finite length, the diffusive motion of the two steps acting like two non-crossing random walkers, as we confirmed in simulations. 


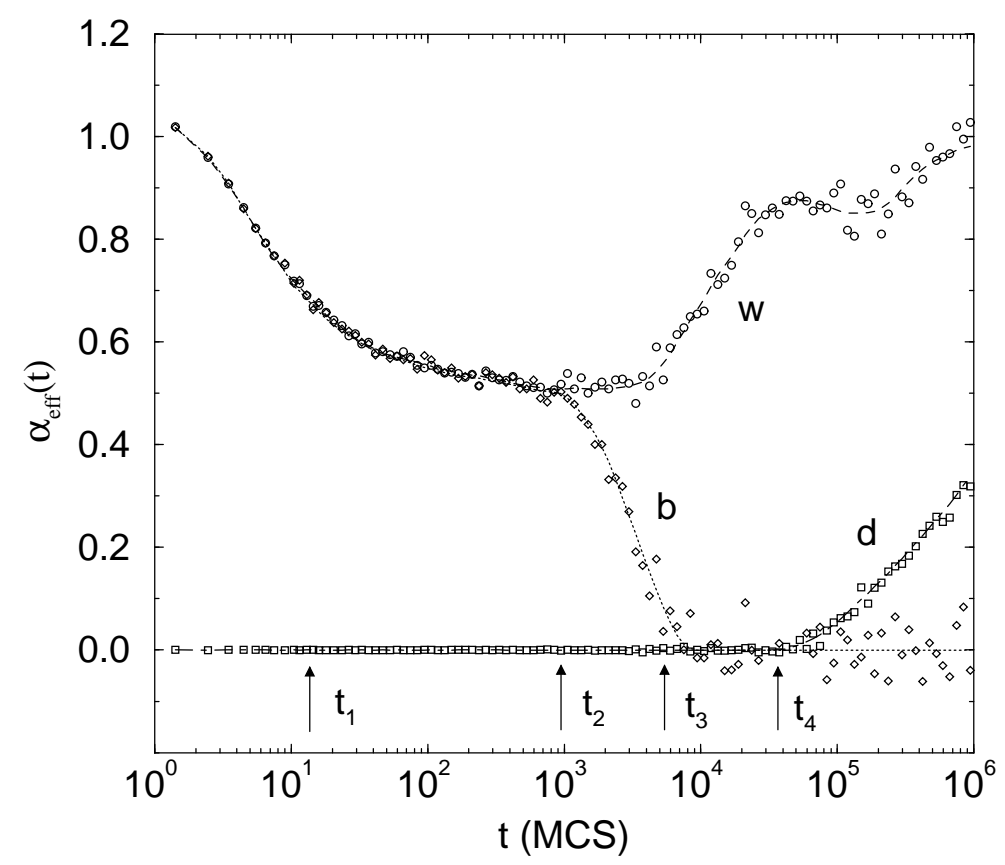

Fig. 2. Effective exponent $\alpha_{\text {eff }}$ for a pair of steps with periodic boundary conditions, using evaporation kinetics, of the non-equilibrium fluctuations $w$ (circles), the width $b$ (diamonds), and the distance between the steps $d$ (squares). Steps of length $L=128$, with $d_{0}=60$, at $k_{B} T / \epsilon=1.0$, averaging over 18,000 realizations, were simulated. The lines are guides to the eye.

(ii) For step-edge diffusion, the average position of each step is conserved. Equilibrium correlation functions are much suitable to describe the dynamics of the pair of steps [21]. In particular, we computed, doing dynamic averaging,

$$
C_{k l}(x, t)=\left\langle\left(h_{k}\left(i+x, \tau_{m}+t\right)-h_{k}^{(0)}\right)\left(h_{l}\left(i, \tau_{m}\right)-h_{l}^{(0)}\right)\right\rangle_{i, \tau_{m}}
$$

with $k$ and $l$ denoting either the same step (the 'intra-step-correlation function' $C_{11}=C_{22}=C(x, t)$ ) or different steps (the 'inter-step-correlation function' $C_{12}=C_{21}=C_{s}(x, t)$ ), where $i$ and $i+x$ are step sites. Averages were taken over several reference times $\tau_{m}$ in equilibrium.

Intra- and intercorrelation functions have been studied before in the framework of a continuum (or Langevin) description, dealing mainly with terrace diffusion [21]. In the s-d case, $C_{s}$ has been argued to vanish; this suggestion may be viewed with care, as indicated by our simulational findings for the discrete SOS model. 


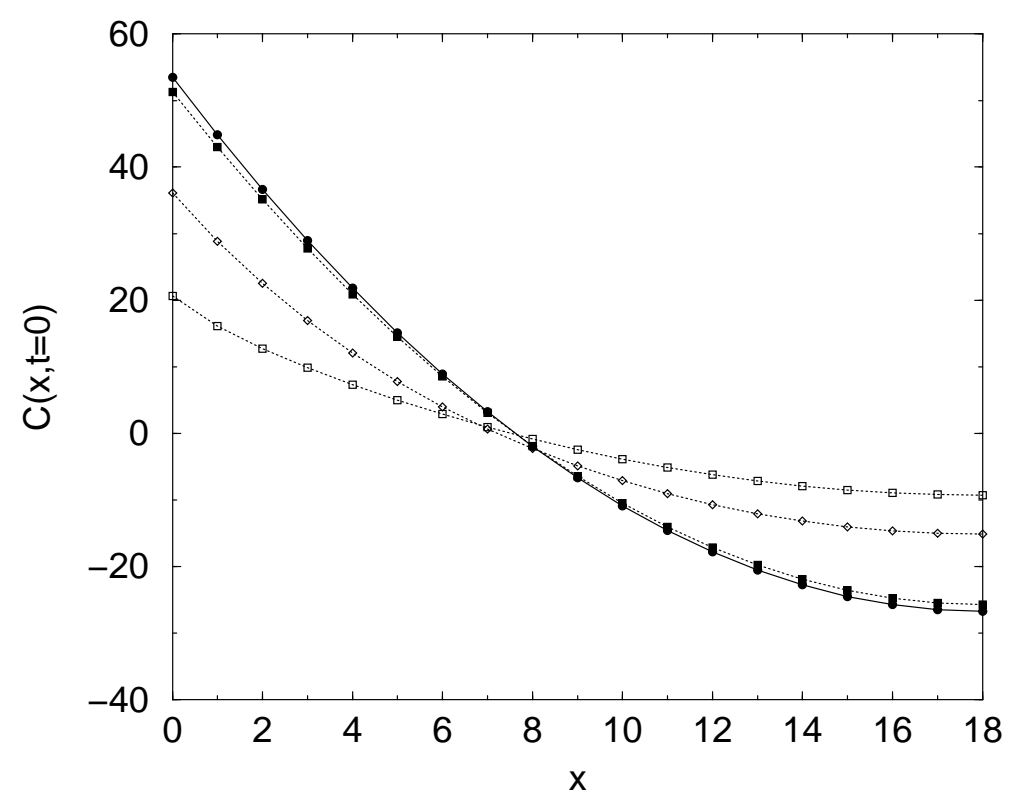

Fig. 3. Equilibrium intra-step-correlations $C(x, t=0)$, for isolated (full symbols) and a pair of steps with separation distance $d_{0}=5$ (open squares) and 15 (open diamonds), using step-edge diffusion. The full circles refer to the continuum expression, eq. (7). Steps of length $L=36$, at $k_{B} T / \epsilon=3.0$ were simulated, using dynamic averaging over, at least, $10^{6}$ reference times $\tau_{m}$.

In our simulations, we varied the step length, $10 \leq L \leq 48$, the separation distance, $2 \leq d_{0} \leq 30$, and temperature $0.8 \leq k_{B} T / \epsilon \leq 3.0$. In addition, the intracorrelation function $C$ for isolated steps was computed.

At $t=0$, intracorrelations, $C(x, t=0)$, for an isolated step were observed to be close to the continuum expression [21]

$$
C(x, t=0)=\frac{L}{2 \Sigma} c(x / L)
$$

with $c(x / L)=1 / 6-x / L+(x / L)^{2}$, see Fig. 3 .

The rather slight deviations between the simulational data and the continuum description may be mainly attributed to the $L$-dependence in front of the scaling function $c$; the deviations diminish for longer steps.

In the presence of the second step, we observe an overall reduction of the intracorrelations compared to those of an isolated step as long as the separation distance $d_{0}$ is not much larger than the width, measured by $b_{s}=\sqrt{b(t=\infty)}$, of each step. The reduction increases monotonically with decreasing $d_{0}$. In the limit of a pair of close-by steps, $d_{0}=2$, the correlation function approximates 
the form $C(x, 0) \propto L c(x / L)$, reflecting the synchronization of the fluctuations of the two steps. However, for larger separation of the steps, the shape of $C(x, 0)$ does not scale perfectly with $x / L$, albeit it does not deviate much, see Fig. 3, from the parabolic shape of the isolated step, Eq. (7). Indeed, we do not expect a scaling behaviour, because, at fixed temperature and separation distance $d_{0}, b_{s}$ and thence the 'overlap' of the fluctuations of the two steps depend clearly on the step length $L$.

The form of the intercorrelation function at $t=0, C_{s}(x, t=0)$, is found to be, at small separation distance $d_{0}$, close to that of corresponding intracorrelation function $C(x, 0)$, reflecting again the synchronization of the fluctuations of the two steps. The shape of $C_{s}$ changes towards a more (co-) sinusoidal form as $d_{0}$ increases. Note that the correlations $C_{s}$ tend to increase with $d_{0}$, as long as the separation distance is comparable to the width of steps, $b_{s}$. They decrease drastically when further enlargening $d_{0}$, because then the two steps only rarely encounter each other.

As time proceeds, both the intra- and intercorrelations are described by, as may be obtained, e.g., from Fig. 4,

$$
C_{(s)}(x, t)=A \cos (2 \pi x / L) \exp (-\gamma t)
$$

where the prefactor $A$ and the exponent $\gamma$ depend on the type of correlations (intra or inter) and the specifics of the steps (temperature, step length, and separation distance). For instance, we find, for $C$ and $C_{s}, \gamma \propto 1 / L^{4}$, with a rather weak (if at all) dependence on $d_{0}$. The exponential decay, with the observed $L$-dependence of the exponent, and the sinusoidal form may be explained by applying Mullins' theory on the flattening of surface corrugations [22] to the steps. Indeed, the validity of that theory in describing the healing of perturbations in one-dimensional SOS models with Kawasaki dynamics has been checked before using Monte Carlo techniques [23].

According to Mullins' theory, for steps of length $L$ with periodic boundary conditions, each step configuration, as it occurs at reference time $\tau_{m}$, will eventually decay exponentially in time, with the relaxation time increasing like $L^{4}$ (in the s-d case), and it will take on a sinusoidal shape with the wavelength $L[22]$. The correlation functions, $C(x, t)$ and $C_{s}(x, t)$, indeed, show just this behaviour. The time needed to reach the asymptotics depends, especially, on the content of other harmonics in the reference configuration.

\section{Summary}




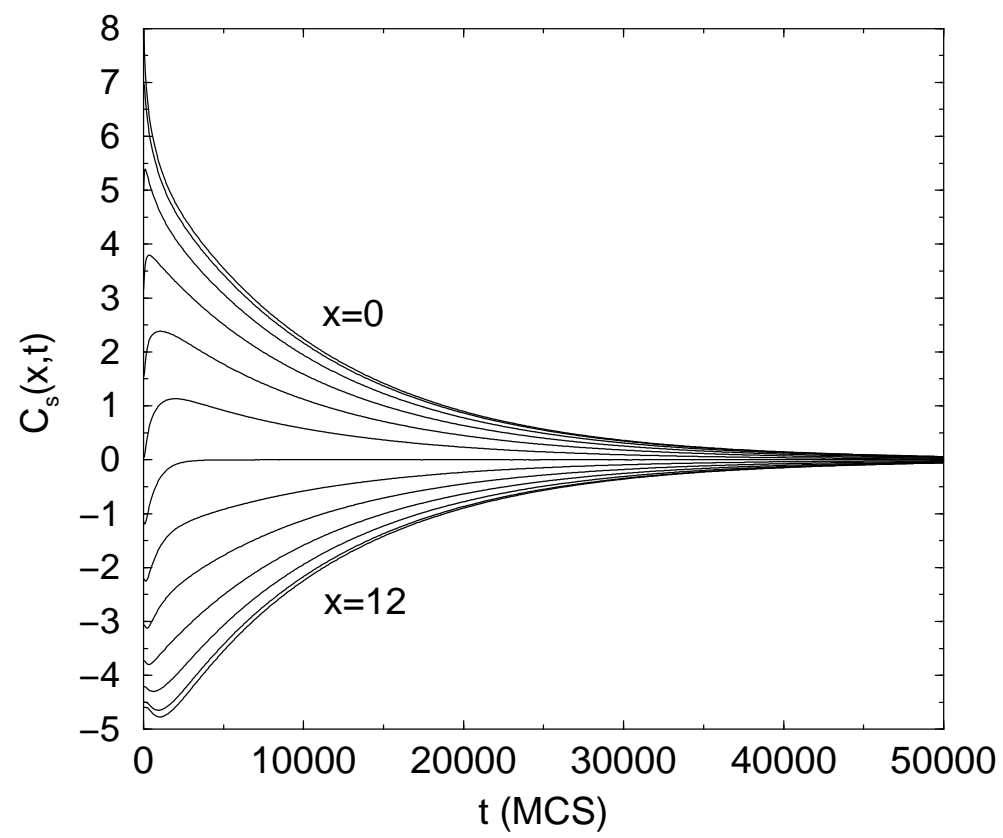

Fig. 4. Inter-step-correlations $C_{s}(x, t)$ for a pair of steps, using step-edge diffusion, with $L=24$ and at $k_{B} T / \epsilon=3.0$, averaging over 200,000 reference times $\tau_{m}$. The different curves refer, from top to bottom, to $x=0,1,2, \ldots, L / 2$. Note that Eq. (8) is approached closely after about 10,000 Monte Carlo steps (MCS).

Using Monte Carlo techniques, the dynamics of isolated steps and pairs of steps of monoatomic height has been studied for two types of kinetics, evaporationcondensation as well as step-edge diffusion, in the framework of SOS models. Periodic and pinned boundary conditions are applied.

For isolated steps, in addition to the asymptotic behaviour at late stages, as obtained from Langevin (or continuum) descriptions, we observe interesting phenomena at short times, including the superdiffusive step fluctuations in the case of evaporation kinetics and a non-monotonic time dependence in the effective exponent in the case of step-edge diffusion with periodic boundary conditions.

For pairs of steps, in the evaporation case, various time scales are identified where distinct processes govern the step dynamics. At late times, applying periodic boundary conditions, the two roughened steps act like two non-crossing random walkers. In the case of step-edge diffusion, results of a recent continuum theory have been checked and extended. In particular, the time-dependent correlation functions along each step and between the steps are non-zero, with their long-time behaviour being described by the classical theory of Mullins on the flattening of surface corrugations above the roughening transition temperature. 


\section{Acknowledgements}

We should like to thank M. Bisani, H. P. Bonzel, P. M. Duxbury, and J. Hager for useful discussions and information. F. Sz. thanks the Deutsche Forschungsgemeinschaft, under grant number SE 324/3-1, and the Hungarian Scientific Research Fund, under grant number OTKA D32835, for financial support.

\section{References}

[1] L. Kuipers, M. S. Hoogeman, and J. W. M. Frenken, Phys. Rev. Lett. 71 (1993) 3517.

[2] M. Giesen-Seibert, R. Jentjes, M. Poensgen, and H. Ibach, Phys. Rev. Lett. 71 (1993) 3521.

[3] M. Giesen-Seibert, F. Schmitz, R. Jentjes, and H. Ibach, Surf. Sci. 329 (1995) 47.

[4] H. C. Jeong and E. D. Williams, Surf. Sci. Rep. 34 (1999) 175.

[5] J. T. Li, R. Berndt, and W. D. Schneider, Phys. Rev. Lett. 76 (1996) 1888.

[6] N. C. Bartelt, T. L. Einstein, and E. D. Williams, Surf. Sci. 312 (1994) 411.

[7] B. Blagojevic and P. M. Duxbury, in: Dynamics of Crystal Surfaces and Interfaces, Eds. P. M. Duxbury and T. Pence (Plenum, New York, 1997), p. 1.

[8] S. V. Khare and T. L. Einstein, Phys Rev. B 57 (1998) 4782.

[9] A. Pimpinelli, J. Villain, D. E. Wolf, J. J. Metois, J. C. Heyraud, I. Elkinani, and G. Uimin, Surf. Sci. 295 (1993) 143.

[10] W. Selke and M. Bisani, in: Anomalous Diffusion, Eds. R. Kutner, A. Pekalski, and K. Sznajd-Weron (Springer, Berlin, 1999), p. 298.

[11] M. Bisani and W. Selke, Surf. Sci. 437 (1999) 137.

[12] B. Swartzentruber, Y. W. Mo, R. Kariotis, M. G. Lagally, and M. B. Webb, Phys. Rev. Lett 65 (1990) 1913.

[13] D. B. Abraham and P. J. Upton, Phys. Rev. B. 39 (1989) 736.

[14] J. D. Weeks, in: Ordering in Strongly Fluctuating Condensed Matter Systems, Ed. T. Riste (Plenum, New York, 1980), p. 293; H. van Beijeren and I. Nolden, in: Structure and Dynamics of Surfaces II, Eds. W. Schommers and P. v. Blanckenhagen (Springer, New York, 1987). 
[15] E. E. Gruber and W. W. Mullins, J. Phys. Chem. Solids 28 (1967) 875.

[16] K. Binder and D. W. Heermann, Monte Carlo Simulation in Statistical Physics (Springer, Berlin, 1992).

[17] M. E. Fisher, J. Stat. Phys. 34 (1984) 667.

[18] G. Forgacs, R. Lipowsky, and Th. M. Nieuwenhuizen, in: Phase Transitions and Critical Phenomena, Vol. 14, Eds. C. Domb and J. L. Lebowitz (Academic Press, London, 1992), p. 135.

[19] H. P. Bonzel and A. Emundts, Phys. Rev. Lett. 84 (2000) 5804.

[20] J. Hager and H. Spohn, Surf. Sci. 324 (1995) 365; J. Hager, Phys. Rev. B 56 (1997) 12499.

[21] B. Blagojevic and P. M. Duxbury, Phys Rev. E 60 (1999) 1279.

[22] W. W. Mullins, in: Metal Surfaces: Structure, Energetics and Kinetics, Eds. R. Vanselow and R. Howe (Springer, New York, 1963), p. 17.

[23] W. Selke and P. M. Duxbury Z. Physik B 94 (1994) 311; Phys. Rev.B. 52 (1995) 17468. 